Supplementary Materials

\title{
Bioinspired Hybrid Micro/Nano-Structure Composited Membrane with Intensified Mass Transfer and Antifouling for High Saline Water Membrane Distillation
}

Xiaobin Jiang ${ }^{\mathrm{a}}$, Yushan $\mathrm{Shao}^{\mathrm{a}}$, Jin $\mathrm{Li}^{\mathrm{a}}$, Mengyuan $\mathrm{Wu}^{\mathrm{a}}$, Yuchao Niu ${ }^{\mathrm{a}}$, Xuehua Ruan ${ }^{\mathrm{b}}$, Xiaoming Yan ${ }^{\mathrm{b}}$, Xiangcun $\mathrm{Li}^{\mathrm{a}}$, Gaohong $\mathrm{He}^{\mathrm{a}, \mathrm{b}^{*}}$ a State Key Laboratory of Fine Chemicals, School of Chemical Engineering, Engineering Laboratory for Petrochemical Energy-efficient Separation Technology of Liaoning Province, Dalian University of Technology, Dalian 116024, P. R. China b School of Chemical Engineering at Panjin, Dalian University of Technology, Panjin 124221, P. R. China

\section{SUPPLEMENTARY METHODS}

\section{Experimental materials.}

Polypropylene (PP) flat microporous membranes with about $0.1 \mu \mathrm{m}$ pore diameter were generously provided by Institute of chemistry, Chinese academy of sciences, Beijing. Silane coupling agent 3-Glycidyloxypropyltrimethoxysilane (KH-560, AR degree, $97 \%$ ) and 1H,1H,2H,2H-perfluorodecyltriethoxysilane (PFDTS) (96 \%) were purchased from Aladdin. Ethanol (AR degree) was supplied by Sinopharm Chemical Reagent Co., Ltd. (China). Tetraethyl orthosilicate (TEOS, AR degree) was purchased from Tianjin Kemiou Chemical Reagent Co., Ltd. (China), and ammonia solution $\left(\mathrm{NH}_{3} \cdot \mathrm{H}_{2} \mathrm{O}, 25 \%\right)$ and Sodium chloride $(\mathrm{NaCl})$ were from Tianjin Damao.

\section{SUPPLEMENTARY RESULTS AND DISCUSSION}

Hybrid micro/nano-structure constructed membrane.

\footnotetext{
* Corresponding author: e-mail: hgaohong @dlut.edu.cn, Phone: +86-411-84986291, Fax: +86-411-84986291
} 
Plasma treatment and surface modification were synchronously used to fabricate targeted superhydrophobic micro/nano-structure on commercial PP membranes. The fabrication procedure was shown in Figure 1a. It is notable that plasma treatment under optimal conditions can effectively enlarge the pore size and graft moieties on original PP membranes, which provide sufficient active site for further modification. To introduce hybrid micro/nano-structure, the membrane surface was loaded by micro/nanoscale $\mathrm{SiO}_{2}$ nanoparticles which were grown to the optimized diameters in sequence. Finally, the typical hydrophobic modification of the micro/nano-structure was achieved via the fluorination of $1 \mathrm{H}, 1 \mathrm{H}, 2 \mathrm{H}, 2 \mathrm{H}$-perfluorodecyltriethoxysilane (PFDTS). Figure 1b clearly illustrated how the interfacial structure varied during the membrane fabrication. The $\mathrm{PP} / \mathrm{N}$ membrane after optimized plasma treatment possesses enlarged surface pore size and enhanced hydrophilicity due to pore expansion effect and generated amino group attributed to the plasma etching (shown in supplementary material of Figure S1 and S2). After fluorination process, the resulting F-nmSiO2-PP/N membrane still exhibits a micro-nano gradient structure (mean size of micro particle is around $0.4 \mu \mathrm{m}$; mean size of nano particle is around $50 \mathrm{~nm}$ ) and sufficient pore size on the membrane surface, leading to reinforcement of the superhydrophobicity (water contact angle, $\mathrm{WCA}=160.49^{\circ}$ ) and regular interfacial roughness with $\mathrm{Ra}=155.0 \mathrm{~nm}$ (shown in supplementary material of Figure S3 and S4). As a comparison, the WCA of F-PP/N (just fluorination on PP membrane), F-nSiO2$\mathrm{PP} / \mathrm{N}$ (fluorination with nano scale sphere loaded) and $\mathrm{F}-\mathrm{mSiO}_{2}-\mathrm{PP} / \mathrm{N}$ (fluorination with micro scale sphere loaded) all ranged around $142^{\circ}$ to $144^{\circ}$ (shown in Figure S4). As shown in Figure 1c and Table S1, compared to original PP membrane, the occurred $\mathrm{O} 1 \mathrm{~s}$ peaks and increased nitrogen content of the PP/N membranes manifested that the amino/imino moieties had been completely grafted after plasma treatment. In Figure 1d, 
with respect of $\mathrm{mSiO}_{2}-\mathrm{PP} / \mathrm{N}$ membrane and $\mathrm{nmSiO}_{2}-\mathrm{PP} / \mathrm{N}$ membrane, the characteristic peaks of $\mathrm{Si}-\mathrm{O}-\mathrm{Si}$ and $-\mathrm{OH}$ moieties on the surface of $\mathrm{SiO}_{2}$ particles appeared at around $1100 \mathrm{~cm}^{-1}$ and $3290 \mathrm{~cm}^{-1}$ respectively, confirming the successful construction of $\mathrm{SiO}_{2}$ structure. The feature peaks of C-F appearing at $1148 \mathrm{~cm}^{-1}$ and $1205 \mathrm{~cm}^{-1}$ derived from $\mathrm{CF}_{2}$ and $\mathrm{CF}_{3}$ functional groups through fluorination step. In consistent with the results of ATR-FTIR spectra, XPS analysis was also monitored as shown in Figure 1c. The Si $2 \mathrm{p}$ and $\mathrm{F} 1 \mathrm{~s}$ peaks were detected on the modified membranes surface, which further proved the formation of covalent bonds after coating and fluorination procedure.

\section{SUPPLEMENTARY FIGURES}
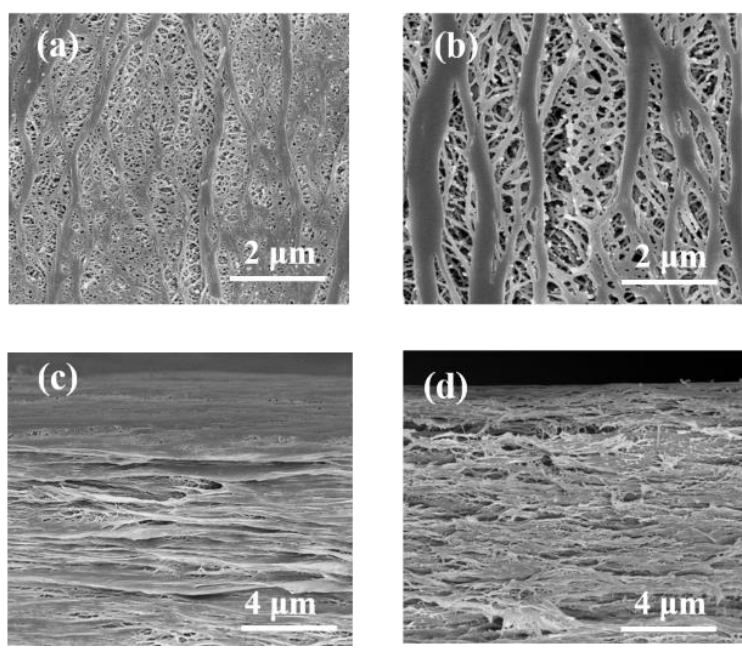

Figure S1. SEM images of the surface and cross section of PP and plasma treated PP/N membrane. (a) PP membrane surface. (b) PP/N membrane surface. (c) PP membrane cross section. (d) PP/N membrane cross section. 


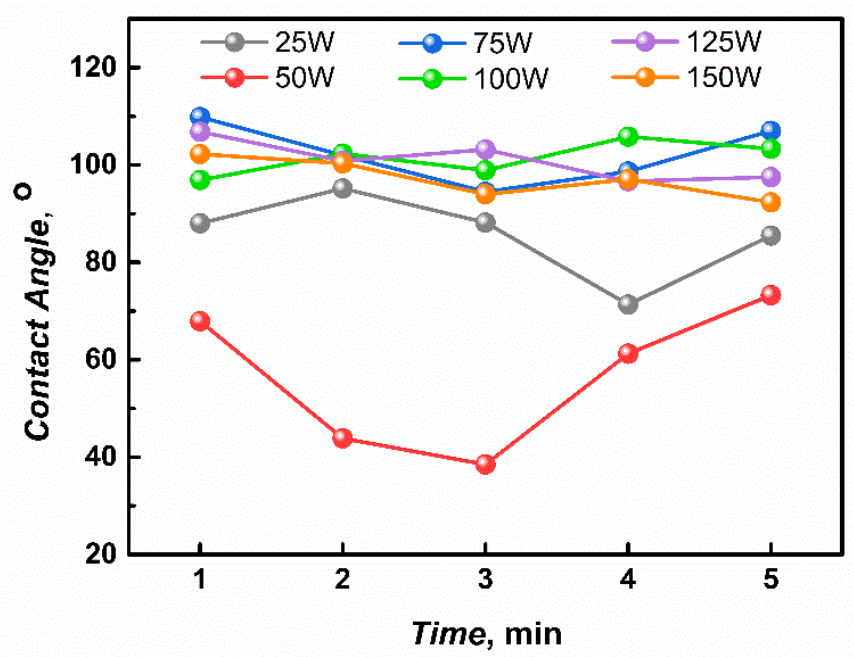

Figure S2. Static water contact angle of $\mathrm{PP} / \mathrm{N}$ membrane after different plasma treatment conditions.

The effect of plasma treating time and power on membrane wettability was systematically evaluated via investigating surface static WCA data. It was found that the wettability of the membranes exhibited dramatical transformation from hydrophobic to hydrophilic after treated by plasma process when exposed to the power of $50 \mathrm{~W}$ for 3 minutes in comparison to other operating conditions, indicating the successful grafting of amino and imino moieties on the membrane surface thus realizing the increased hydrophilicity. 

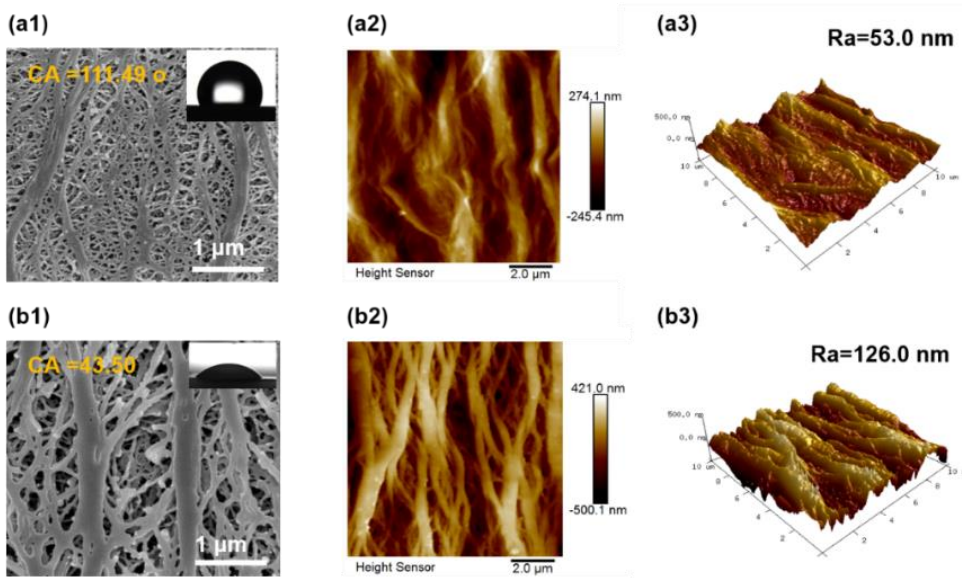

(b3)

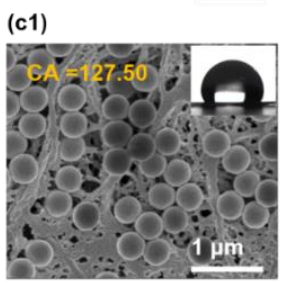

(c2)
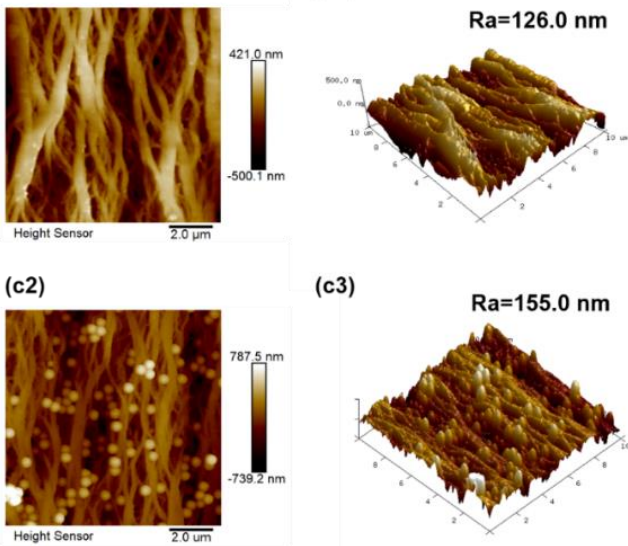

(c3)
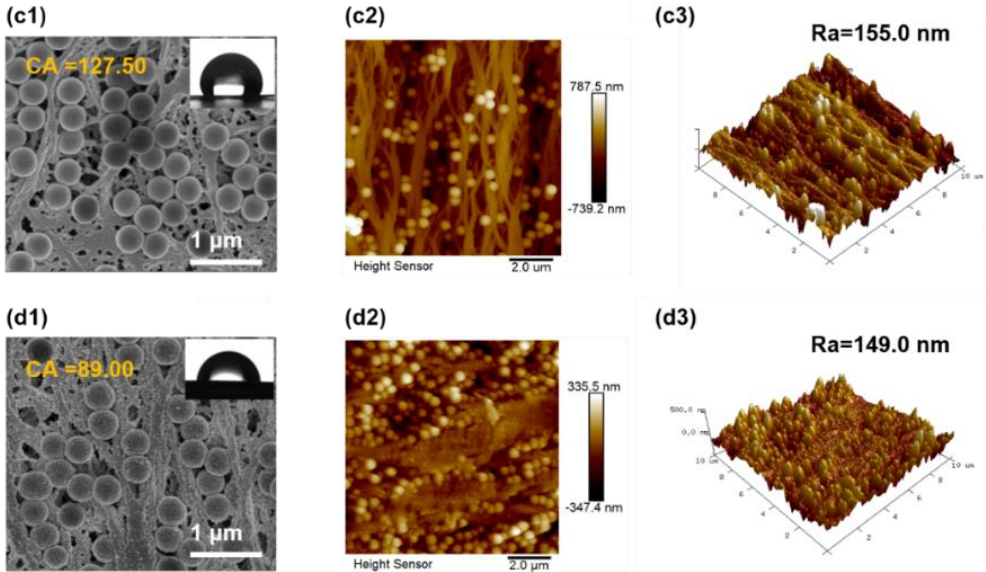

(d2)

(d3)
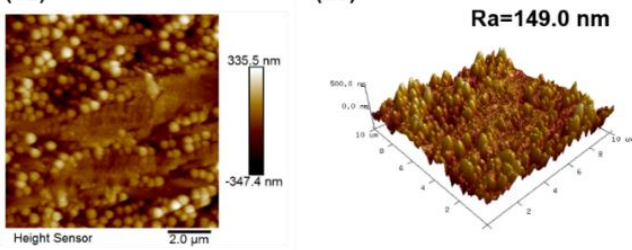

(e2)
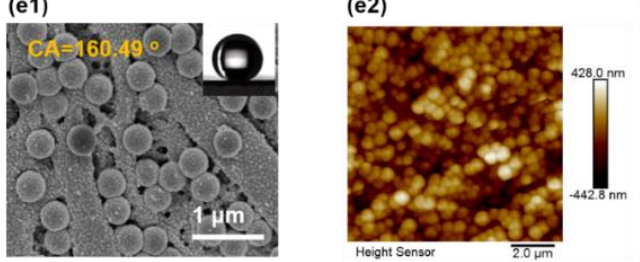

(e3)

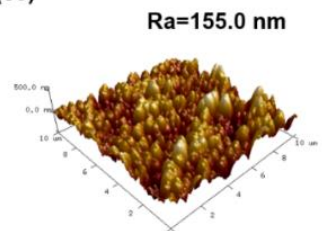

Figure S3. SEM images and water contact angle (WCA) and AFM (2D and 3D) images of different membranes (a1, a2, a3) original PP, (b1, b2, b3) PP/N after plasma treatment, (c1, c2, c3) mSiO2 $-\mathrm{PP} / \mathrm{N},(\mathrm{d} 1, \mathrm{~d} 2, \mathrm{~d} 3) \mathrm{nmSiO}_{2}-\mathrm{PP} / \mathrm{N},(\mathrm{e} 1, \mathrm{e} 2$, e3) $\mathrm{F}-\mathrm{nmSiO} 2-\mathrm{PP} / \mathrm{N}$.

(a)

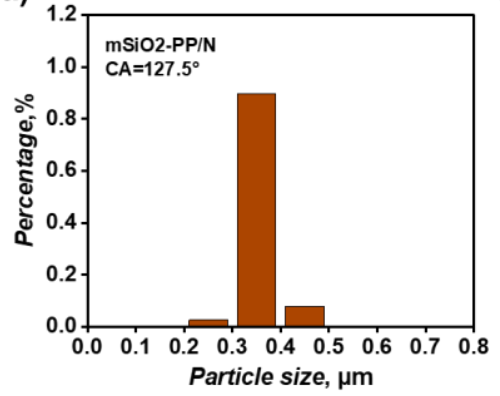

(b)

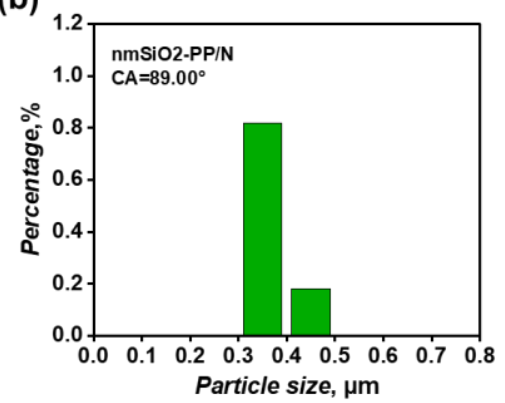

(c)

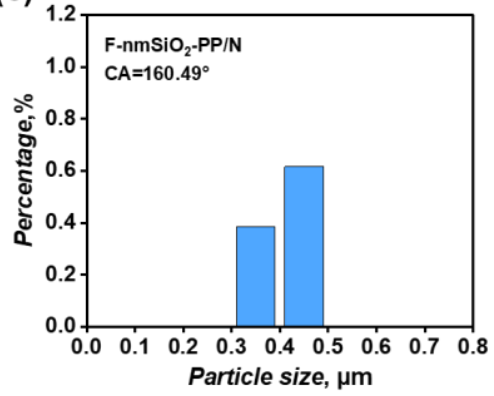


Figure S4. The micro particle size distribution of membranes. (a) $\mathrm{mSiO}_{2}-\mathrm{PP} / \mathrm{N}$ membrane; (b) $\mathrm{nmSiO}_{2}-\mathrm{PP} / \mathrm{N}$ membrane; (c) $\mathrm{F}-\mathrm{nmSiO}_{2}-\mathrm{PP} / \mathrm{N}$ membrane
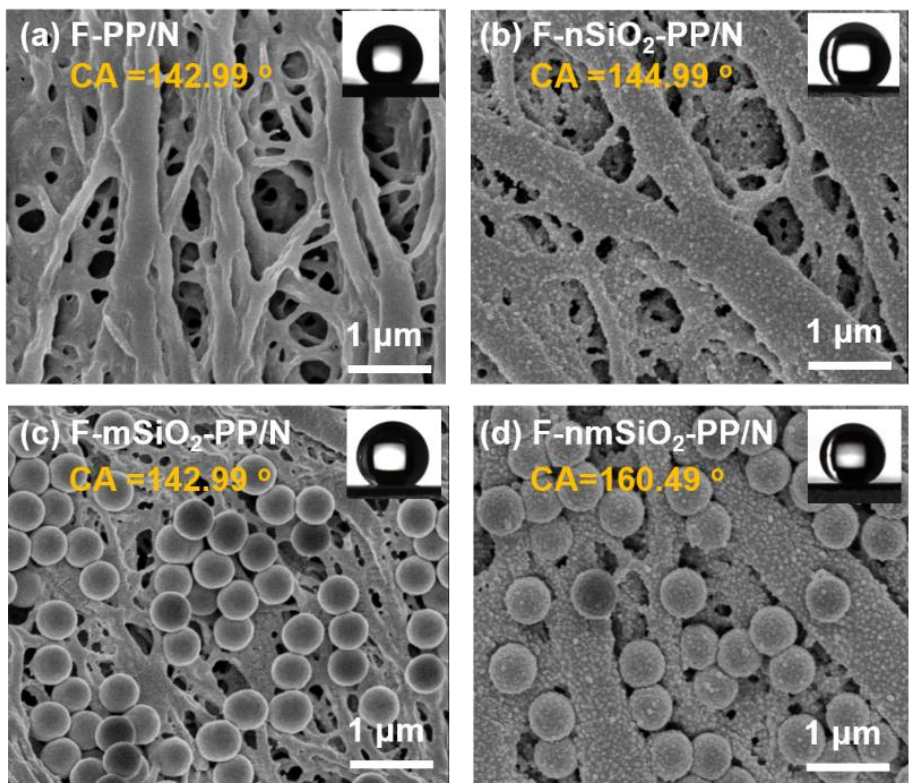

Figure S5. Comparison of morphology, wettability of the membranes with different structure.(A) SEM and WCA of (a) F-PP/N. (b)F-nSiO2-PP/N. (c) F-mSiO $2-P P / N$. (d) $\mathrm{F}-\mathrm{nmSiO}_{2}-\mathrm{PP} / \mathrm{N}$.

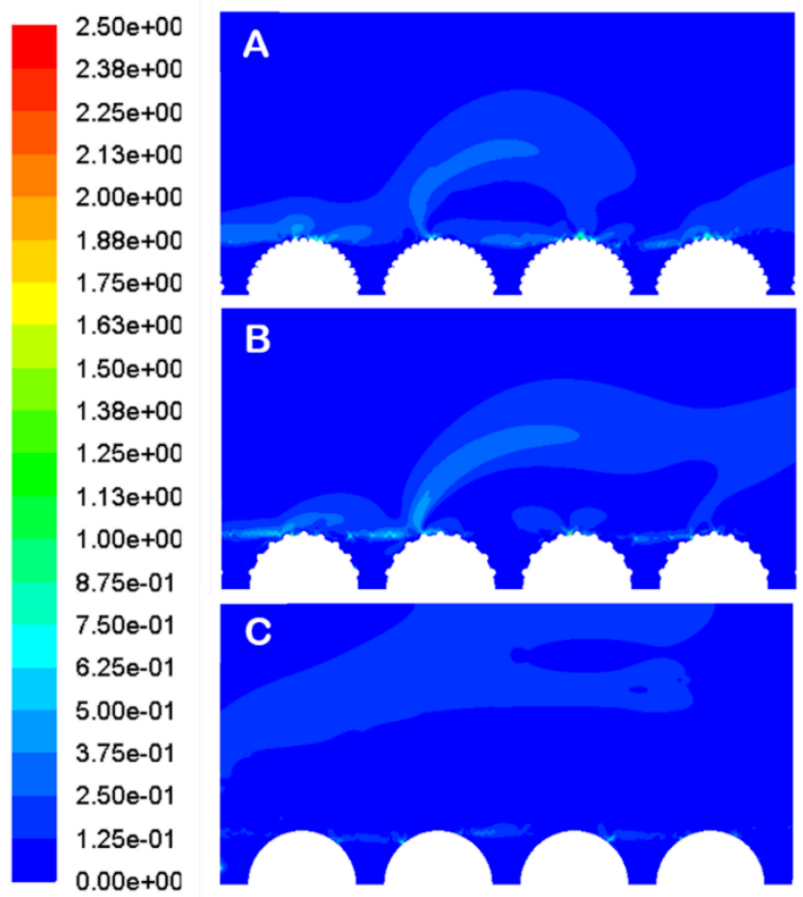

Figure S6. Simulated velocity distribution on the corresponding nano-micro $\mathrm{SiO}_{2}$ particle surface (a) F-nmSiO $2-\mathrm{PP} / \mathrm{N}$ (Full loaded). (b) $\mathrm{F}-\mathrm{nmSiO} 2-\mathrm{PP} / \mathrm{N}$ (half loaded) (c) 
$\mathrm{F}-\mathrm{mSiO}_{2}-\mathrm{PP} / \mathrm{N}$.
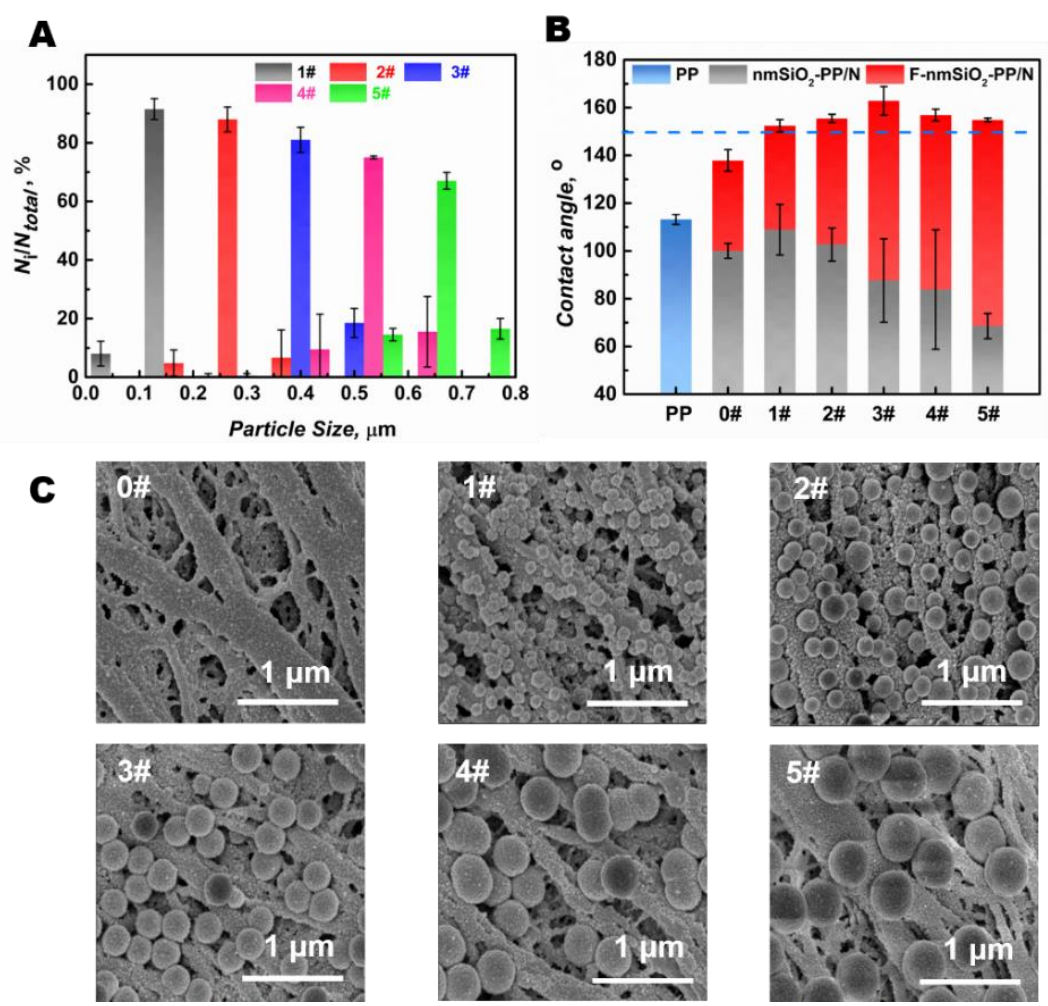

Figure S7. Characteristics of the modified membranes loaded by $\mathrm{SiO}_{2}$ particles with different diameters. (a) The micro particle size distribution. (b) WCA of the fabricated membranes. (c) Morphology comparison. (the membranes codes from $1 \#$ to $5 \#$ represent the membranes consist of microparticles with increased diameters, and $0 \#$ is the membrane only loaded by nanoparticles.) 


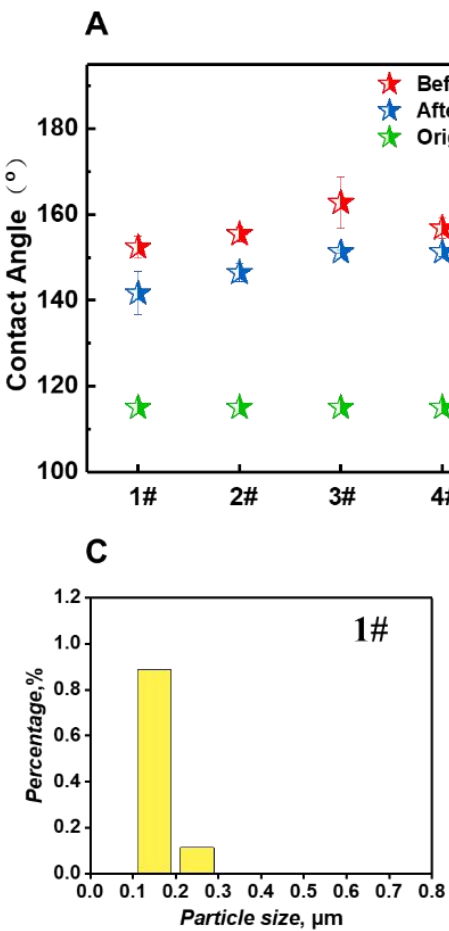

B
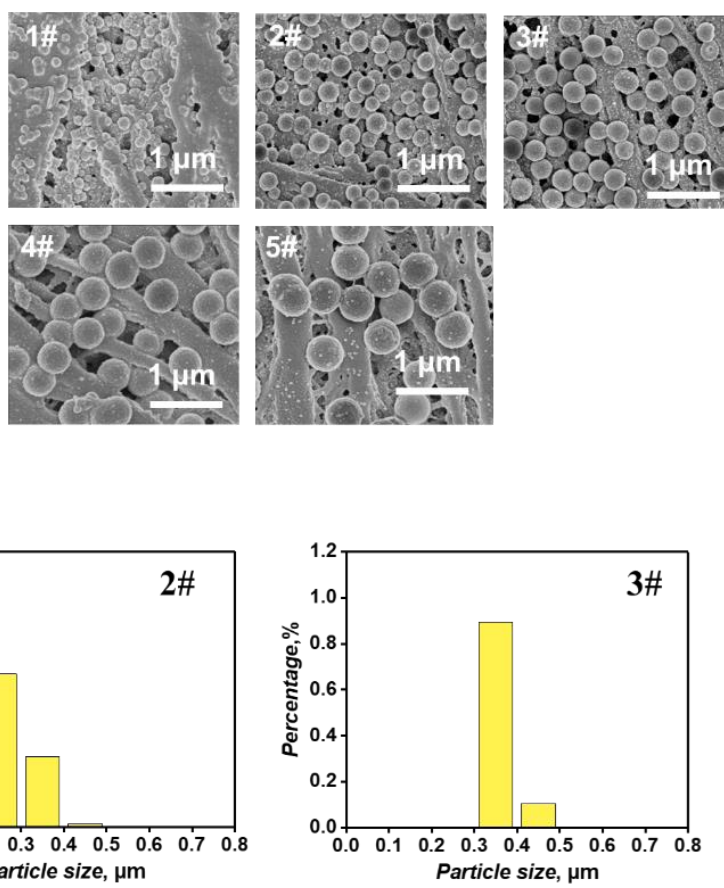

Particle size, $\mu \mathrm{m}$
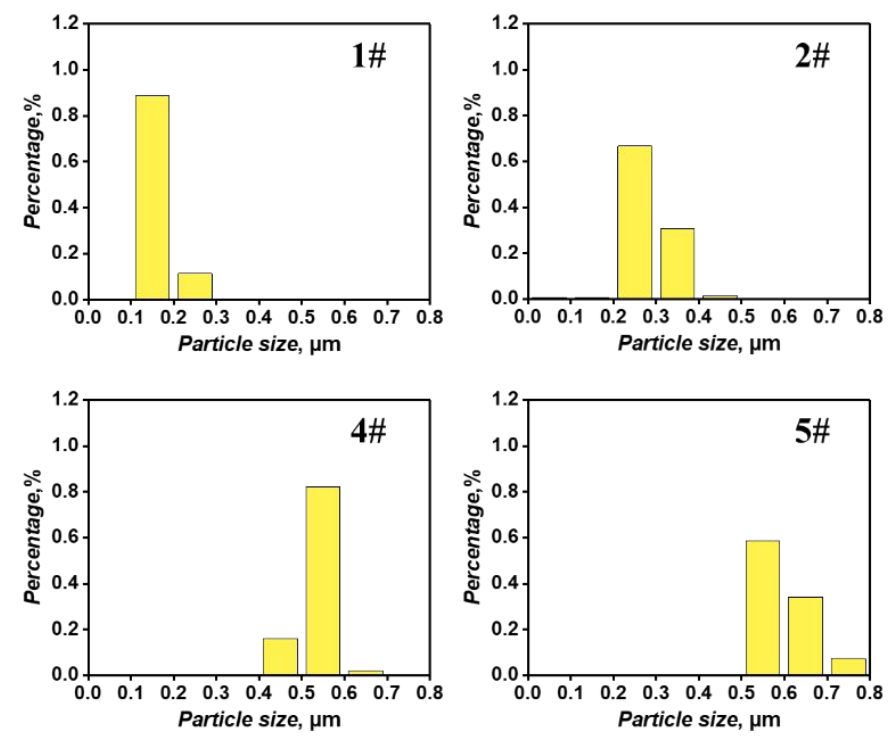

Figure S8. Wettability (a), SEM images (b) and the micro particle size distribution (c) of the membranes before and after MD tested in water bath under $80^{\circ} \mathrm{C}$ and stirring at $250 \mathrm{r} / \mathrm{min}$ for $150 \mathrm{~h}$. (Membranes named from $1 \#$ to $5 \#$ represent the membranes consist of microscale structures with increased diameters of $0.13,0.26,0.37,0.43,0.55 \mu \mathrm{m}$ respectively). 


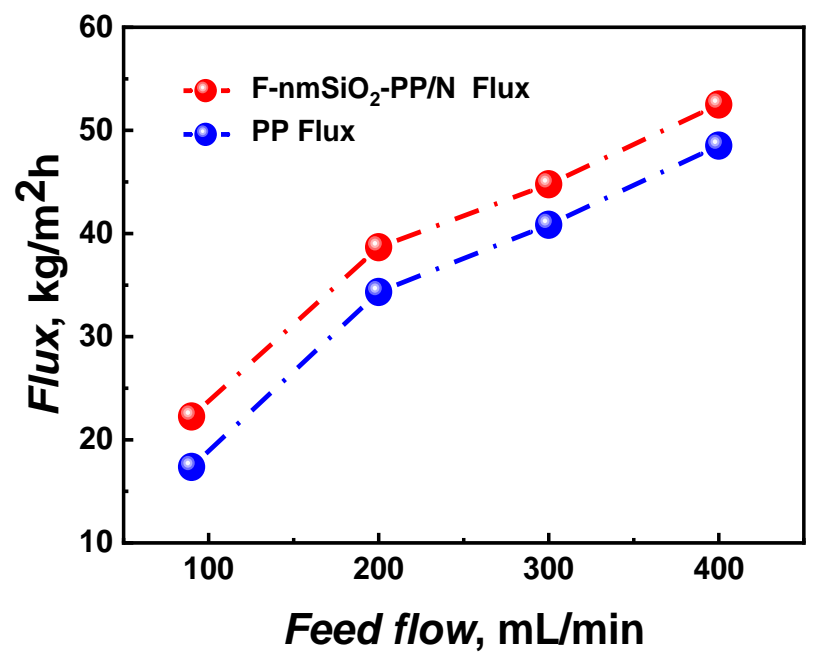

Figure S9. Effect of different feed rate on the permeate flux of original PP and F$\mathrm{nmSiO} 2-\mathrm{PP} / \mathrm{N}$ membranes. (3.5 wt $\% \mathrm{NaCl}$ solution; $\triangle \mathrm{P}: 0.096 \mathrm{MPa}$; feed temperature: $\left.80{ }^{\circ} \mathrm{C}\right)$

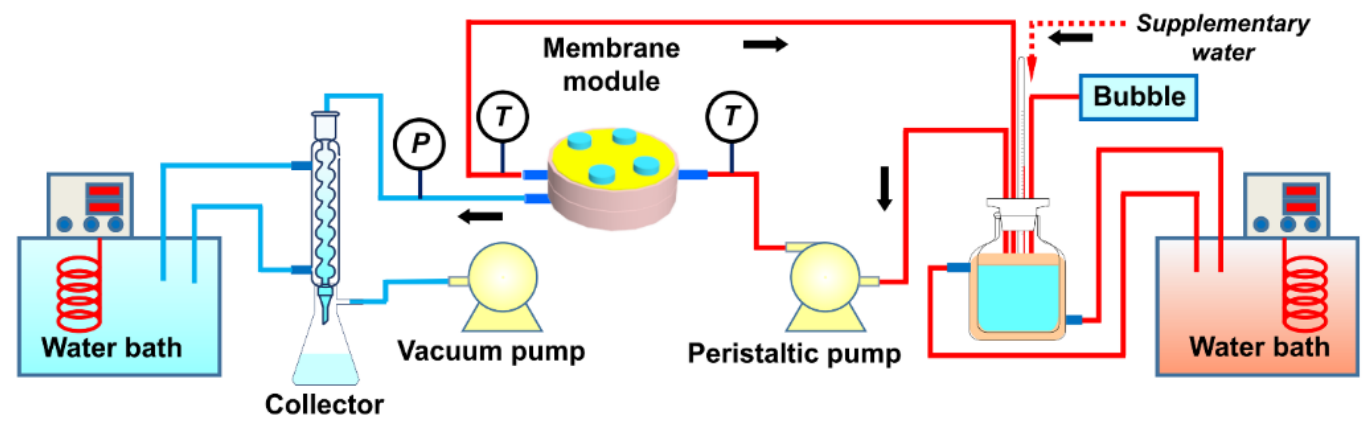

Figure S10. Schematic diagram of VMD experimental setup.

Table S1. Surface composition (atom.\%) of the membranes before and after modification.

\begin{tabular}{llllll}
\hline & $\mathrm{PP}$ & $\mathrm{F}-\mathrm{PP} / \mathrm{N}$ & $\mathrm{F}-\mathrm{mSiO}_{2}-\mathrm{PP} / \mathrm{N}$ & $\mathrm{F}-\mathrm{nmSiO} 2-\mathrm{PP} / \mathrm{N}$ & $\mathrm{F}-\mathrm{nmSiO}_{2}-\mathrm{PP} / \mathrm{N}$ \\
\hline $\mathrm{C} 1 \mathrm{~s}$ & $98.55 \%$ & $81.20 \%$ & $63.61 \%$ & $58.60 \%$ & $35.79 \%$ \\
$\mathrm{O} 1 \mathrm{~s}$ & $1.45 \%$ & $16.69 \%$ & $23.20 \%$ & $26.62 \%$ & $12.62 \%$ \\
$\mathrm{~N} 1 \mathrm{~s}$ & --- & $2.11 \%$ & $1.62 \%$ & $1.00 \%$ & $0.65 \%$ \\
$\mathrm{Si} 2 \mathrm{p}$ & --- & --- & $11.58 \%$ & $13.78 \%$ & $7.13 \%$ \\
$\mathrm{~F} 1 \mathrm{~s}$ & --- & --- & --- & --- & $43.79 \%$ \\
\hline
\end{tabular}

